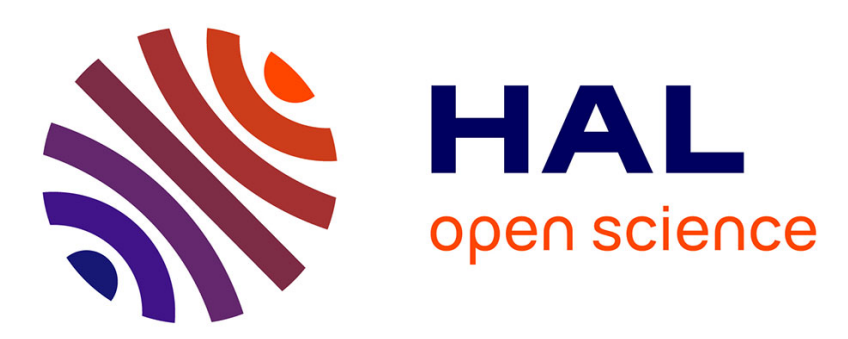

\title{
Sur l'interpretation énergétique du champ moléculaire et l'aimantation paramagnétique
}

\author{
Pierre Weiss
}

\section{To cite this version:}

Pierre Weiss. Sur l'interpretation énergétique du champ moléculaire et l'aimantation paramagnétique. J. Phys. Radium, 1930, 1 (1), pp.1-10. 10.1051/jphysrad:01930001010100 . jpa-00205406

\section{HAL Id: jpa-00205406 https://hal.science/jpa-00205406}

Submitted on 1 Jan 1930

HAL is a multi-disciplinary open access archive for the deposit and dissemination of scientific research documents, whether they are published or not. The documents may come from teaching and research institutions in France or abroad, or from public or private research centers.
L'archive ouverte pluridisciplinaire HAL, est destinée au dépôt et à la diffusion de documents scientifiques de niveau recherche, publiés ou non, émanant des établissements d'enseignement et de recherche français ou étrangers, des laboratoires publics ou privés. 


\title{
LE JOURNAL DE PHYSIQUE
}

\author{
LE RADIUM
}

\section{SUR L'INTERPRÉTATION ÉNERGÉTIQUE DU GHAMP MOLEGULAIRE ET L'AIMANTATION PARAMAGNÉTIQUE \\ par Pierre WEISS.}

\begin{abstract}
Sommaire. - La condition nécessaire et suffisante pour qu'un paramagnétique obéisse à la loi de Curie généralisée $\chi(\tau-\Theta)=C$ est que son énergie dépende de l'aimantation par le terme $n \sigma^{2} / 2$. Lorsqu'une théorie donne cette loi cela prouve seulement que ce terme énergétique est contenu explicitement ou implicitement dans les hypothèses sur lesquelles elle repose.

La représentation de $1 / \chi T$ en fonction de $1 / T$ se prête à un contrôle serré de la loi de Curie généralisée. A titre d'exemple, elle est appliquée à la critique des expériences précises de Chatillon et de Mlle Serres qui donnenl pour l'ion Co" dans le sulfate de cobalt anhydre les trois moments voisins de $25 ; 2$; 67 et 26 magnétons. On obtient dans ce cas et dans d'autres des droites dans de grands intervalles de température que les théories qui donnent la loi $\chi(\tau-\Theta)=C$ par un développement en série sont insuffisantes à expliquer.

La condition énergétique apprend que dans les solutions à pcint de Curie indépendant de la concentration le porteur de moment ne peut être l'ion tout entier mais une partie seulement qui se déplace par rapport au reste par l'action du champ.
\end{abstract}

Paramagnétisme pur. - Langevin a montré que lorsqu'une substance magnétique a comme les gaz parfaits une énergie fonction de la température seulement et, par conséquent, indépendante de l'état d'aimantation, le principe de Carnot exige que l'aimantation $\sigma$ ne dépende que du quotient du champ $\boldsymbol{H}$ par la température absolue $\boldsymbol{T}$

$$
\sigma=f\left(\frac{H}{T}\right) .
$$

Si l'on se borne aux petites valeurs de la variable $\boldsymbol{H} / \boldsymbol{T}$, il vient:

$$
\sigma=\frac{C}{T} H
$$

où $C$ est la constante de Curie. Ces équations représentent ce que l'on peut appeler le paramagnétisme pur. Le sulfate de gadolinium à 8 molécules d'eau, étudié par Woltjer et Kamerlingh Onnes, est un exemple de substances répondant à l'équation (1). Celles qui sont étudićes seulement dans les limites de la proportionnalité de l'aimantation au champ, et qui satisfont à l'équation (2) sont plus nombreuses. On peut citer l'oxygène aux faibles pressions, l'alun de fer, le sulfate de manganèse à quatre molécules d'eau.

Paramagnétisme à point de Curie. - Conservons, quelle que soít la variation thermique, la désignation de paramagnétiques pour les substances qui premnent une aiman-

Le Journal de physique et le radiun. - Série vil. - T. I. - No 1. ¿ JaNVier $193^{\circ}$. 
tation faible et proportionnelle au champ. On constate que la plupart d'entre elles obéissent, non à la loi de Curie (2) mais à la loi plus générale :

$$
\sigma=\frac{C}{T-\Theta} H
$$

où $C$ est une constante dont nous montrerons plus loin la signification et où la température $\Theta$ porte le nom de Point de Curie.

Cette loi a été trouvée d'abord pour les métaux ferromagnétiques au-dessus du Point de Curie. Mais son champ d'application est beaucoup plus étendu. Le palladium et le platine obéissent à cette loi avec des points de Curie virtuels, situés bien au-dessous du zéro absolu. L'oxygène aux grandes densités y satisfait avec une valeur de $\Theta$ négative, proportioanelle à la densité.

Les mesures précises sur le paramagnétisme des sels solides ont montré que, très généralement, ces substances obéissent elles aussi, non à la loi de Curie primitive (2), mais à l'équation (3).

Il a paru surprenant qu'il en fùt de même pour les solutions des ions paramagnétiques. Le fait a été rencontré pour la première fois par Foëx dans les solutions d'azotate de cobalt. Cabrera et Dupérier y ont ajouté la constatation que le Point de Curie des solutions de l'ion Mn" est indépendant de la concentration. Chatillon a trouvé à son tour que les points de Curie de plusieurs solutions de l'ion $\mathrm{Co}^{\prime \prime}$ ne varient pas avec la concentration.

Condition nécessaire et suffisante du paramagnétisme à Point de Curie. Je me propose d'établir que la condition nécessaire et suffisante pour qu'une substance paramagnétique obéisse à la loi $(3)$ est que l'énergie $U$ de cette substance dépende de l'aimantation par le terme:

$$
-\frac{n}{2} \sigma^{2}
$$

où $n$ est une constante, et par ce terme seulement.

Champ moléculaire énergétique. - Puisque nous devons étudier des substances autres que les paramagnétiques purs et dont l'énergie, par conséquent, dépend de l'aimantation, nous introduisons la notation

$$
H_{m}=-\frac{\partial U}{\partial \sigma}
$$

Nous appelons $H_{m}$ le champ moléculaire énergétique pour le distinguer d'un autre champ moléculaire que nous définirons plus loin.

Calculons $I_{m}$ à partir des donnćes expérimentales sur l'aimantation.

Soit $c_{\sigma}$ la chaleur spécifique à aimantalion constante; l'accroissement d'énergie en fonction de $T$ et de $\sigma$ est:

$$
\mathrm{d} U=c_{\sigma} \mathrm{d} T-H_{m} \mathrm{~d} \sigma .
$$

Mais puisque le travail élémentaire communiqué à la substance est:

$$
\mathrm{d} \mathscr{G}=H \mathrm{~d} \sigma
$$

il vient :

$$
\mathrm{d} Q=\mathrm{d} U-\mathrm{d} \mathscr{G}=c_{\sigma} \mathrm{d} T-\left(\boldsymbol{H}+\boldsymbol{H}_{m}\right) \mathrm{d} \sigma .
$$

En écrivant que d $U$ et d $Q / T$ sont différentielles exactes. on a par la combinaison des deux équations ainsi obtenues:

$$
H_{m}=T \frac{\partial H}{\partial T}-H
$$


Condition nécessaire. - En empruntantàl'équation $(3) H$ et $\frac{\partial H}{\partial T}$ en fonction de $T$ et de $\sigma$ et en portant dans (9) on trouve :

$$
H_{m}=\frac{\Theta}{C} \sigma
$$

ou en désignant le quotient des deux constantes $\Theta$ et $C$ par $n$,

$$
H_{m}=-\frac{\partial U}{\partial \sigma}=n \sigma
$$

et par conséquent :

$$
U=-\frac{n \tau^{2}}{2}+f(T)
$$

Définition du champ moléculaire terme correctif de l'équation d'état. Nous allons démontrer la proposition réciproque par une voie détournée, mais qui met bien en évidence la signification des diverses grandeurs utilisées. Prenons comme point de départ le paramagnétisme pur, sans nous limiter à la proportionnalité de l'aimantation au champ et proposons-nous d'apporter à l'équatior (1) .une correction telle qu'elle représente l'aimantation d'une substance quelconque, ferromagnétique par exemple. Nous ajoutous à cet effet au champ extérieur $H$ un terme $h_{m}$. Si $h_{m}$ est une fonction de $\sigma$ et de $T$, n'importe quelle loi peut être ainsi représentée. Nous appelons $h_{m}$ le champ moléculaire correctif de l'équation d'état.

L'équation du paramagnétisme (1), corrigée par $h_{m}$ s'écrit

$$
\sigma=f\left(\frac{H+h_{m}}{T}\right)
$$

ou, en résolvant, par rapport à $H$

$$
H=-h_{m}+T \varphi(\sigma)
$$

et en portant $H$ et $\frac{\mathrm{d} H}{\mathrm{~d} \boldsymbol{T}}$ dans $(9)$, on 'exprime le champ moléculaire énergétique au moyen du champ moléculaire terme correctif :

$$
H_{m}=h_{m}-T \frac{\partial h_{m}}{\partial T} .
$$

Donc, lorsque le champ moléculaire correctif de l'équation d'état $h_{m}$ ne dépend pas de la température, il est égal au champ moléculaire énergétique.

$$
h_{m}=\frac{1}{\frac{1}{T}} \int_{0}^{\frac{1}{T}} H_{m} d \frac{1}{T}
$$

On déduit de (13)

Le champ moléculaire correctif de l'équation d'état est donc la valeur moyenne du champ moléculaire énergétique entre $T$ et $\infty, \frac{1}{T}$ ćtant la variable an moyen de laquelle cette moyenne est prise. II résulte de (14) la proposition réciproque de la précédente :

Si le champ moléculaire énergétique est indépendant de la température, il est égal au champ moléculaire terme correctif de l'équation d'état. 
Condition suffisante. - Nous allons nous appuyer sur cette réciproque pour démontrer la deuxième partie de la proposition. Si l'énergie est donnée par l'équation :

$$
U=-\frac{n \sigma^{2}}{2}+f(T)
$$

on a :

$$
\boldsymbol{H}_{m}=-\frac{\partial U}{\partial \sigma}=n \sigma .
$$

Puisque cette valeur est indépendante de la température, elle est aussi le lerme correctif $h_{m}$ de l'équation d'étal :

$$
\sigma=\frac{C}{T} H
$$

qui devient :

$$
\sigma=\frac{C}{T}(H+n \sigma)
$$

ou

$$
\sigma=\frac{C}{T-C n} H
$$

et avec la notation $C n=\Theta$

$$
\sigma=\frac{C}{T-\theta} H
$$

Il résulte en particulier de cette déduction que la constante $C$ de l'équation (3) est la constante de Curie qu'aurait la substance si, 'sans rien changer par ailleurs, on supprimait l'énergie mutuelle d'orientation.

Donc, quand une théorie donne la loi du paramagnétisme à Point de Curie, cela prouve seulement qu'explicitement ou implicitement les hypothèses contiennent l'énergie magné-

tique $-\frac{n \sigma^{2}}{2}$. Leur vérification ne va pas au delà.

Représentation graphique du paramagnétisme à champ moléculaire. L'équation (3) peut s'écrire en iutroduisant le coefficient d'aimantation $\%=\frac{\sigma}{H}$.

$$
\frac{1}{\%}=\frac{T-\Theta}{C}
$$

ou

$$
\frac{1}{\chi}=\frac{T}{C}-n
$$

L'inverse du coefficient d'aimantation est une fonction linéaire de la température. Cette proposition est équivalente à l'existence d'une énergie de la forme :

$$
U=-\frac{n \sigma^{2}}{2}+f(T)
$$

Les deux propositions sont donc vérifiées ou infirmées en même temps. Elles sont en mème temps ou rigoureuses ou approchées.

Il a élé fait un fréquent usage de la représentation graphique de $1 / \%$ en fonction de $T$. 
On obtient des droites qui donnent par leur coefficient angulaire l'inverse de la constante 4le Curie et qui rencontrent l'axe des températures au point de Curie. La forme (16) montre aussi que le coefficient $n$ est égal à la distance de la droite à l'origine des coordonnées, mesurée parallèlement à l'axe des $1 / \%$.

L'étude de nombreuses substances a donné dans des intervalles étendus des droites (1) avec toute la précision des mesures.

Cependant une exception importante a été signalée. Kamerlingh Onnes et ses collaborateurs ont montré qu'aux basses températures il se produit à partir de la loi linéaire des 'écarts systématiques qu'il a désignés par le terme d'anomalie cryomagnétique. Ces écarts sont plutôt exceptionnels à la température de l'air liquide et fréquents à celle de l'hydrogène liquide. Nous y reviendrons.

A côté de ses avantages, la représentation de $1 / \gamma$ en fonction de $T$ a l'inconvénient de l'emploi d'ordonnées d'autant plus grandes que la quantité mesurée est plus petite et la même erreur relative est représentée par des longueurs très différentes. Elle exige un graphique étendu dans les deux sens pour la représentation de la loi de Curie exacte alors qu'il importe de représenter les écarts de cette loi.

Mais on peut écrire la formule (16) sous la forme:

$$
\frac{1}{\chi^{T}}=\frac{1}{C}-\frac{n}{T}
$$

En portant $\frac{1}{\% T}$, en ordonnées et $\frac{1}{T}$, en abscisses on obtient encore une droite sur laquelle on lit $1 / C$ par l'ordonnée à l'origine et le coefficient $n$ du champ moléculaire par le coefficient angulaire changé de signe. Le Point de Curie s'ensuit par $\Theta=$ C.n.

Dans cette représentation, la loi de Curie exacte est donnée par une parallèle à l'axe des abscisses. Cet axe peut donc être rejeté au-dessous de la figure, de manière à donner une grande importance aux écarts systématiques ou accidentels à partir de la loi régulière.

Tant que les écarts de la loi de Curie sont faibles, une même erreur relative est représentée par une même longueur.

Les moments de $\mathrm{Co}^{\prime \prime}$ dans $\mathrm{SO}^{4} \mathrm{Co}$ anhydre. - Je vais donner des exemples de la loi linéaire et montrer la portée de ce mode de représentation en faisant l'étude critique d'un cas de paramagnétisme particulièrement délicat, la détermination des trois moments très rapprochés que peutprendre $\mathrm{Co}^{\prime \prime}$ dans le sulfate de cobalt anhydre. En effet, $\mathrm{MI}^{\mathrm{He}}$ Serres a découvert deux états de cette substance caractérisés par les moments de $26,0\left(^{2}\right)$ et de $25,6\rceil\left(^{3}\right)$ magnétons. M. Chatillon $\left(^{4}\right)$ a confirmé ces observations et a constaté en outre l'existence du sulfate anhydre à 25 magnétons.

On obtient le sulfate à 26 magnétons par calcination à $60^{\circ}$. L'état à 25,67 magnétons - le sulfate hexagonal d'Etard - résulte de la précipitation par refroidissement du sel dissous dans l'acide sulfurique bouillant. Une fois obtenu, cet état résiste à la calcination

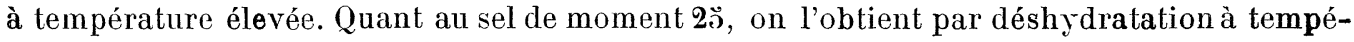
rature modérée, restant au-dessous de $400^{\circ}$.

Les 16 séries d'expériences de $\mathbf{M}^{\text {"le }}$ Serres et de M. Chatillon ont été représentées dans la figure ci-jointe et les résultats qui en ont été déduits sont consignés dans le tableau suivant.

(1) Voir, par exemple, la représentation graphique des mesures sur les terres rares, B. Cabrera, J. de Phys., 6 8., t. 8 (1927), p. 2 et 3.

(2) C. R., t. 181 (1928), p. 714.

(3) Inédit.

$\left({ }^{4}\right)$ Thèse Strasbourg 1927 et Ann. de Phys. 
$\mathrm{CoSO}^{t}$ à 25 magnétons.

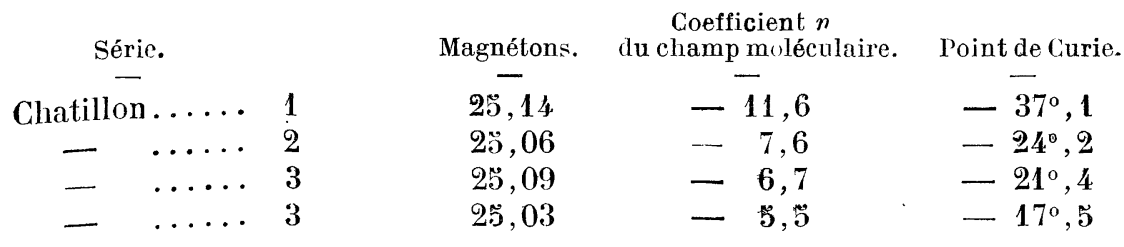

CoSO $0^{4}$ à 20,67 magnétons .

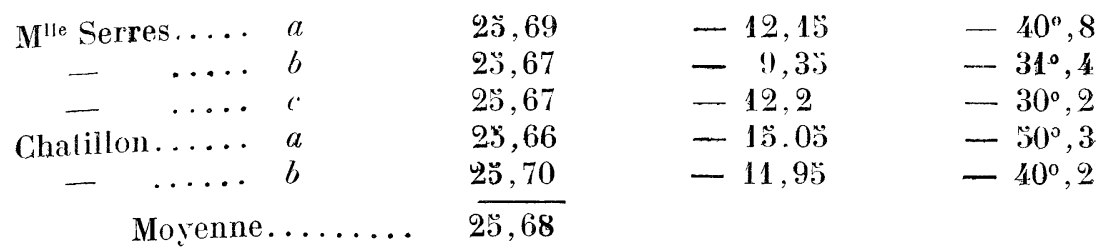

CoSO' à 26 magnétons.

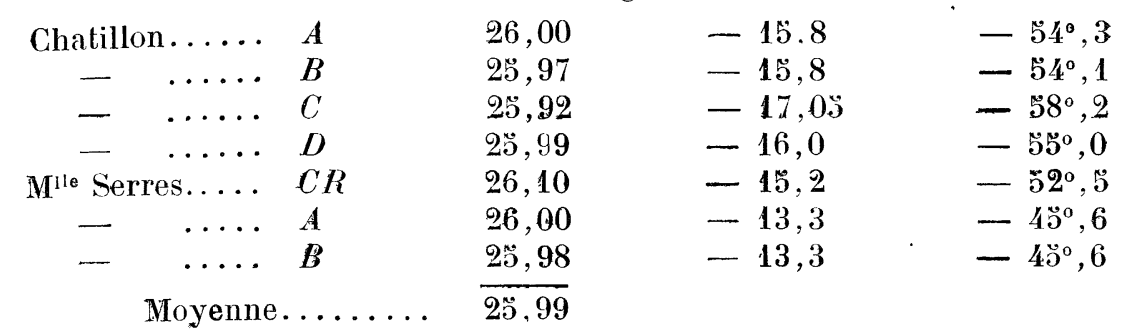

Les moments de ce tableau ne diffèrent jamais que d'un petit nombre de centièmes de magnétons de ceux qui ont été obtemus par les auteurs au moyen de la représentation de $1 / \%$ en fonction de $T$. Les Points de Curic ne diffèrent des leurs que de quantités en général inférieures au degré.

Chacune de ces séries est bien représentée par une droite, les érarts des points observós ne dépassant qu'exceptionnellement 2 pour 1000 . Ces droites se répartissent en trois growpes A, B, C correspondant at modes de traitement que nous avons indiqués. Dans les deux premiers les inclinaixons trè diverses des droites mettent en évidence un fait qui a ćté souvent signalé : des deux constantes $C$ et $\Theta$ e'est la seconde qui, de beaucoup, est la plus sensible à des influences aceidentelles, petites différences dans le traitement de la substance ou impuretés minimes.

Les mesures du groupe A sont ler moins préeises. Elles ont été faites par Chatillon, non pour caraetériser un état nouveau mais pour reconnaître si la substance possède le moment dominant de 23 magnétons, bien connu par de nombreuses séries sur le sulfate hydraté ou en solution el sur des sels d'autres anions. La valeur moyenne du moment donné par le groupe A est de 25,08 magnétons.

Dans le groupe B comprenant trois séries de $\mathbf{M}^{\text {lle }}$ Serres et deux de M. Chatillon, la convergence se produit avec netteté vers la même constante de Curic. La valeur moyemne du moment est de 25,68 magnétons.

Le groupe $\mathrm{C}$ comporte trois séries de $\mathrm{M}^{1 \mathrm{le}}$ Serres et quatre de $\mathbf{M}$. Chatillon. Ici non seulement les constantes de Curie sont voisines mais les Points de Curie sont trop peu différents pour séparer les droites. Je les ai décalées les unes par rapport aux autres parallèlement à l'axe des abscisses. Les moments sont tous compris dans la bande hachurée représentant $26 \pm 0,1$ magnétons et sont pour la plupart (voir fig. 1) étroitement groupés autour de 26,0. La moyenne donne 2:,99 magnétons. 


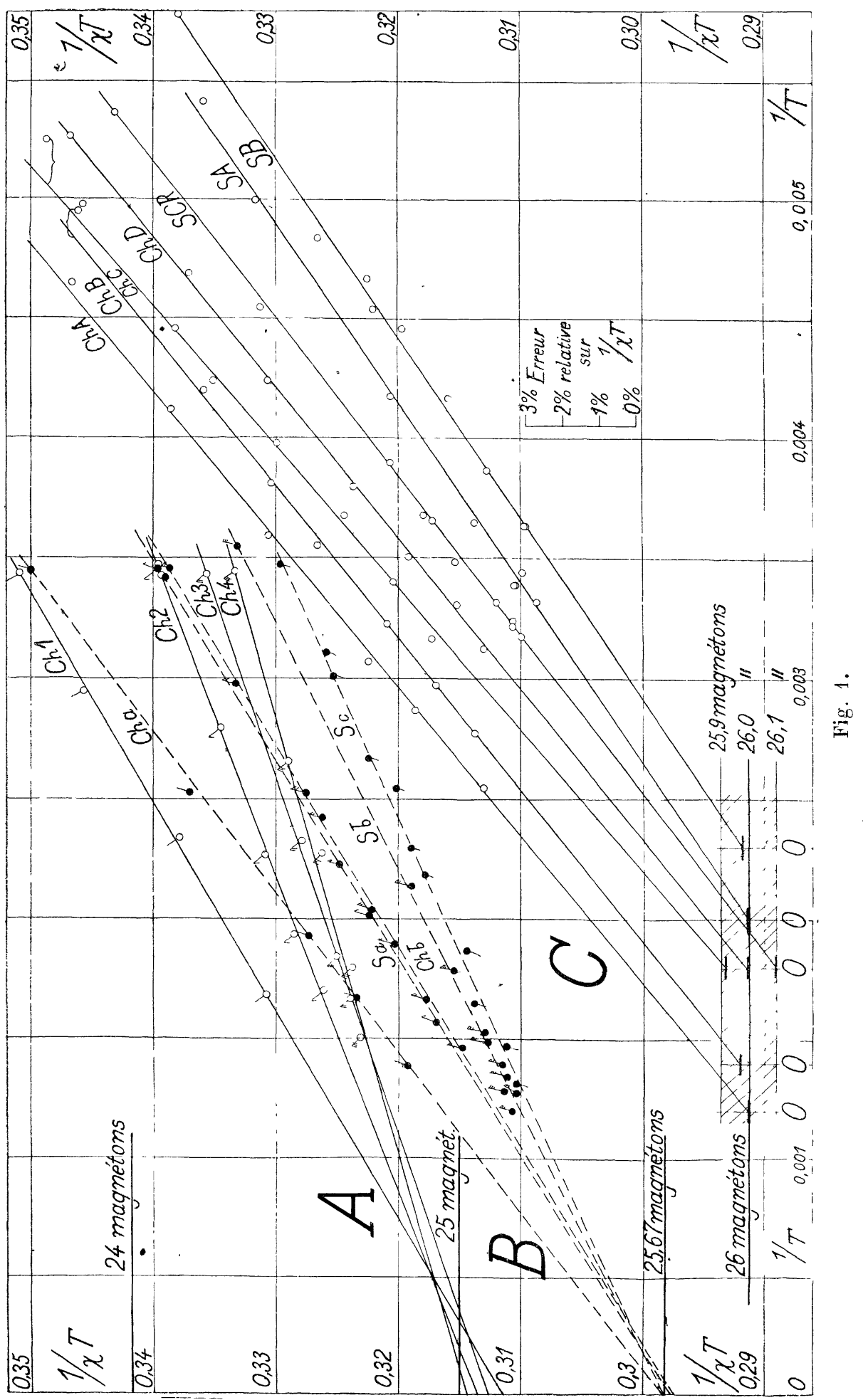


Les substances calcinćes à haute tempéralure de ce dernier groupe passent jour ètro amorphes. Une des séries cependant (la série $A$ de $M^{\text {lle }}$ Serres) provient d’un sulfate cristallisé en octaèdres.

La critique rigoureuse à laquelle la représentation en $1 / \% T$ et $1 / T$ nous a permis de soumettre les expériences confirme donc l'existence des trois moments caractéristiques de trois états différents du $\mathrm{Co}^{\prime \prime}$ dans le sulfate anhydre.

Les théories du champ moléculaire. - Qualitativement on peut trouver un méca-

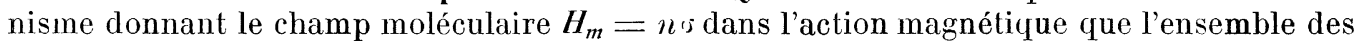
porteurs de moments élémentaires, atomes, ions ou molécules, exercent sur l'un d'entre eux en vertu de la loi de Coulomb. Mais on voit facilement $\left(^{1}\right)$ que cet effet est beaucoup trop faible pour donner les Points de Curie du fer, du nickel, du cobalt. On peut tourner la difficulté en admettant qu'au moment magnétique élémentaire se superpose un moment électrostatique, un dipôle de même direction que lui $\left({ }^{2}\right)$. Le champ moléculaire est alors un langage magnétique pour exprimer des forces en réalité électrostatiques.

La grandeur des moments électrostatiques qui sont nécessaires pour reproduire le champ moléculaire des ferromagnétiques est du mème ordre que celle de tous les moments de dipôles électriques comnus par ailleurs. Elle est à plus forte raison suffisante pour reproduire les champs moléculaires des paramagnétiques.

Mais cette hypothèse électrostatique, tout comme l'hypothèse magnétique primitive, ne donne que les champs moléculaires et les Points de Curie positifs. Les Points de Curie négatifs restent inexpliqués $\left(^{3}\right)$.

Debye et Cabrera et Palacios (') ont tenté d'expliquer le champ moléculaire des paramagnétiques à partir de propriétés atomiques. Dans la théorie de Debye les moments atomiques sont attribués à des orbites électroniques subissant la quantification d'orientation par rapport à la direction du champ. Le nombre des orbites d'une certaine orientation est réglé par la loi de répartition de Maxwell-Boltzmann. L'énergie qui intervient dans l'application de cette loi est, outre celle du champ extérieur, une énergie potentielle attribuée à chaque orientation quantique. Le résultat de Debye (ses équations 20 et $21^{\prime}$ ), transcrit avec nos notations est :

$$
\chi=\frac{C}{T}\left(1+\frac{\Theta}{T}+\frac{A}{T^{2}}+\ldots .\right) .
$$

$\left(C=\mathrm{C}^{\mathrm{t}} \mathrm{e}\right.$ de Curie, $\Theta$, $A$ constantes $)$

équation qui peut aussi s'écrire :

$$
\frac{1}{\chi T}=\frac{1}{C}\left(1-\frac{\Theta}{T}+\frac{a}{T^{2}}+\ldots . .\right) \text {. }
$$

Dans la théorie de Cabrera et Palacios l'ensemble des orbites momentogènes qui donne un moment atomique constant peut prendre un nombre fini d'orientations par rapport à des axes liés à la matière : le réseau cristallin ou l'atome lui-même $\left(^{5}\right)$.

A chaque orientation du moment correspond une certaine énergie potentielle. Le résultat de cette théorie est aussi un développement en série de la forme (17) ou (18). Si on les arrête au deuxième terme on retrouve la loi linéaire du paramagnétisme à champ moléculaire avec $\Theta$ comme Point de Curie.

(1) P. Weiss, Ann. de Phys., 9e s., t. 1 (1914), p. 134.

(2) P. Weiss, C. R., t. 178 (1924), p. 739. DebYe, Handbuch der Radiologie, t. 6 (1925), p. 703.

(3) Deby Quantentheorie. Phys. Z., t. 27 (1926), p 67. Cabrera, J. de Phys., 6• s., t. 8 (1927), p. 257. - Cabrera et Palacios, Ann. Soc. Esp. Fis. y Quim., t 24 (1928), p. 293.

(4) Loc. cit.

(5) Cette théorie contient ainsi, sous une autre forme, l’idée de l'entraînement de la direction privilégiée de quantification par l'agitation thermique, due à E. Baurr et celle de la liaison de la direction privilégiée au réseau cristallin (P. Weıss, J. de Phys., s. VI. t. 4 (1923), p. 153. Weiss et Foëx, Le Magnétisme, p. 173). 
Et en effet on se rend compte facilement que les hypothèses faites conduisent, pour l'énergie d'aimantation, à un développement dont le premier lerme est de la forme - $n \sigma^{2} / 2$. Ces théories admettent aussi bien les Points de Curie négatifs en l'absence de tout renseignement sur le signe de l'énerg ${ }_{1}$ e intra-atomique, sur laquelle elles reposent. Elles donnent d'ailleurs lieu à des difficultés. Celle de Debye ne donne pas entre la constante de Curie et

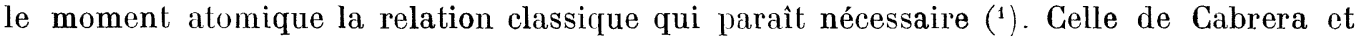
Palacios ne donne le deuxième terme que gràce à une dissymétrie de l'énergie pour deux orientations opposées du moment. Mais cette dissymétrie entraîne aussi une différence entre la $\mathrm{C}^{\text {to }}$ de Curie, donnée par la théorie, et sa valeur classique et, pour la saturation absolue, une valeur moindre que celle qui correspond au parallélisme complet des aimants élémentaires. Si l'on admet, comme cela paraìt nécessaire, que les différences sont négligeables, $\Theta$ tend aussi vers zéro.

Mais je me propose surtout de montrer qu'une théorie qui ne donne le Point de Curie que par le deuxième terme d'un développement en série, ne rend compte des faits que d'une manière insuffisante. La forme (18) montre que si l'on fait usage de la représentation en $1 / \% T$ et $1 / T$ l'ordonnée à l'origine est égale à $1 / C$ et le coefficient angulaire de la tangente au point $1 / T=0$ est $-\Theta / C$, la suite du tracé étant en général curviligne en vertu des termes d'ordre plus élevé. Çes théories ne renseignent donc en aucune façon sur le caractère de la courbe ailleurs qu'à l'origine et les longues droites données avec une haute précision par l'expérience, sont entièrement inexpliquées. Elles exigent que l'énergie d'aimantation se réduise à $n \sigma^{2} / 2$ avec une grande approximation. Le phénomène physique qui donne cette énergie reste à trouver.

Si l'on veut faire rentrer les phénomènes cryomagnétiques dans une représentation de la forme (18), il faut avoir recours à un développement qui exprime la rapidité extrême, avec laquelle ces phénomènes disparaissent aux températures croissantes pour faire place à la loi linéaire exacte.

Sur le paramagnétisme phénomène moléculaire, atomique ou intra-atomique. - Langevin dans sa théorie du paramagnétisme qui s'applique en première ligne aux gaz, considérait des molécules libres douées de moments.

Plus récemment Debye $\left({ }^{2}\right)$ a rassembléles raisons pour lesquelles l'aimantation ne peut être un phénomène moléculaire, mais doit ĉtre attribuée à un mécanisme intra-atomique. On sait que la constante diélectrique variable avec la température résulte en général de l'orientation, par le champ électrique, de molécules douées de dipôles et est par conséquent fondée sur un mécanisme analogue à celui de la théorie du paramagnétisme de Langevin. Si la polarisation paramagnétique reposait elle aussi sur la rotation des molécules, on devrait, quand elles portent à la fois un moment magnétique et un dipôle électrostatique, obtenir une aimantation par le champ électrique ou une polarisation diélectrique "par le champ magnétique. Toutes les tentatives faifes pour observer ces effets ont donné des résultats négatifs.

La polarisation paramagnétique se produit dans des substances cristallisées telle que le sulfate de gadolinium aux températures les plus basses c'est-à-dire, dans des conditions où il n'y a aucune possibilité de rotation de la molécule.

La fusion qui donne la liberté de rotation aux molécules et produit fréquemment un changement important de la constante diélectrique 'n'a en général qu'une influence subordonnée sur le coefficient d'aimantation.

On peut ajouter à ces raisons un nouvel argument tiré de la relation entre l'énergie d'aimantation et l'existence d'un point de Curie différent du zéro absolu. Nous avons déjà rappelé que contrairement à ce que la théorie de l'orientation moléculaire faisait supposer, les solutions des ions de la famille du fer n'obéissent pas à la loi de Curie, mais à la loi plus générale des paramagnétiques à champ moléculaire. Le fait a été constaté par G. Foëx $\left({ }^{3}\right)$,

(1) P. Wriss et R. Forrer, $C . R$, t. 186 (1928), p. 821.

(2) Paramagnetische Magnetisierung und Orientierungshypothèse.

(3) Thèse Strasbourg (1921) et Ann. de Plyys., t. 16 (192!), p. 174. 
sur des solutions d'azotate de cobalt et de sulfate ferreux ammoniacal. Cabrera et Duperier ( ${ }^{1}$ ) ont trouvé que le Point de Curie des solutions de l'ion Mn est indépendant de la concentration. Châtillon $\left({ }^{2}\right)$ a confirmé cette indépendance pour les solutions du chlorure, du sulfate et de l'azotate de coball.

Il faut donc que l'énergie de ces substances dépende de l'aimantation par un terme non nul, égal à $n \sigma^{2} / 2$, où $n$ ne varie pas avec la concentration.

Considérons en particulier les solutions très étendues. Si l'ion comprenant l'atome magnétique et le cortège d'atomes non magnétiques qui l'entoure forme un tout rigide, il est impossible de concevoir que l'énergie dépende de son orientation dans le dissolvant. Si par contre le moment n'est lié rigidement qu'à une partie de l'ion et peut se mouvoir par rapport au poids mort formé de l'autre partie, ce déplacement relatif peut fort bien être accompagné d'un changement d'énergie. Le porteur de moment n'est qu'une partie de l'ion, et la configuration de celui-ci change par aimantation. Une déformation quasi-élastique entre le porteur du moment et le reste de l'ion provoquée par le champ, correspondrait à une énergie positive - $n \sigma^{2} / 2$ du système, avec une valeur négative de $n$ ot un Point de Curie situé au-rlessous du zéro absolu.

Quand le ehamp moléculaire est indépendant de la concentration on doit admettre que le mécanisme énergétique est situé dans la proỉondeur de l'atome ou de l'ion, le manière à ne pas être influencé par le voisinage des autres porteurs de moment. Lorsque, comme dans le cas de l'oxygène, le coefficient du champ moléculaire et avec lui la distance du Point de Curie au zéro absolu sont proportionnels à la densité, l'accumulation d'énergie doit se produire entre les porteurs voisins de moments et augmenter avec le nombre les porteurs contenus dans une sphère d'action autour de l'ion considéré.

(1) J. de Phys., (6 s., t. 6 (1925), p. 121.

(2) Thèse Strasbourg (192i) et Ann. de Phys., t. 9 (1928), p. 18 i. 